\title{
UDC 582.4
}

SCOPUS CODE 2101

https://doi.org/10.36073/1512-0996-2019-4-95-100

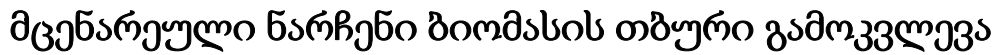

mossm xusogsmodo

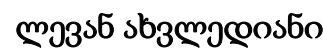

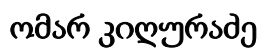

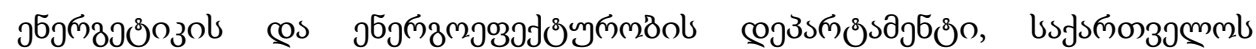

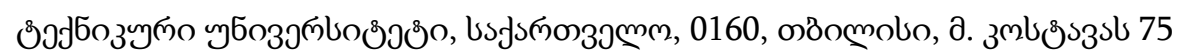
E-mail: japaridzeotari@gmail.com

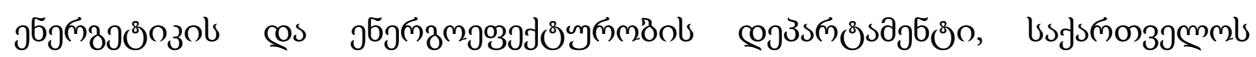

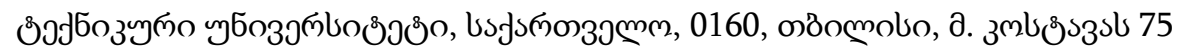
E-mail: samixada1995@gmail.com

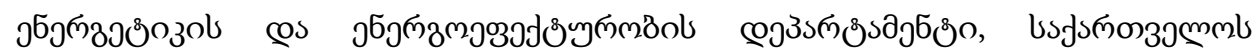

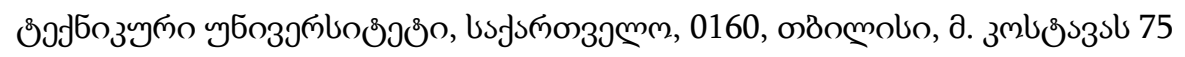
E-mail: kiguradzeomar@gmail.com

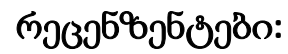

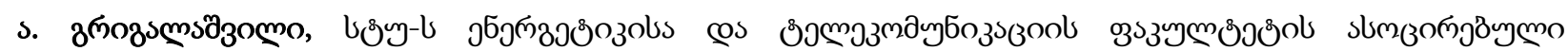

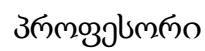

E-mail: a.grigalashvili@gtu.ge

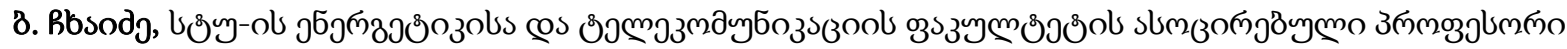

E-mail: bckaidze@yahoo.com

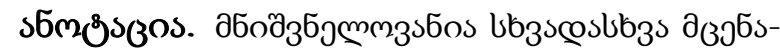

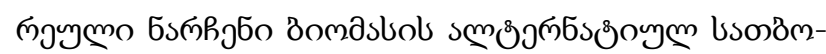

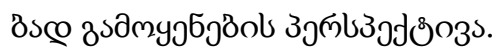

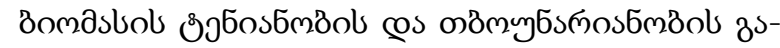

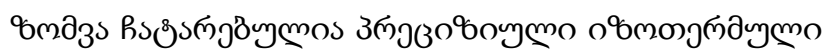

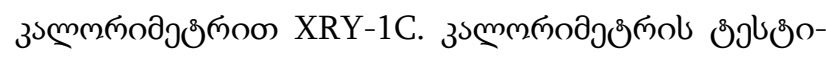

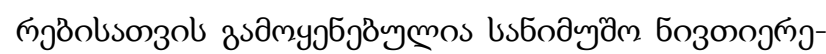

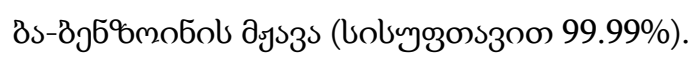

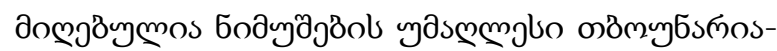

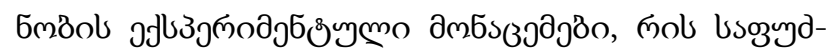

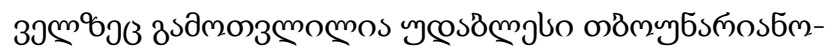

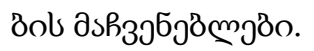

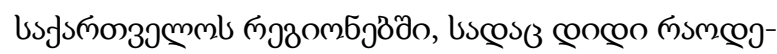

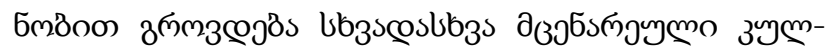

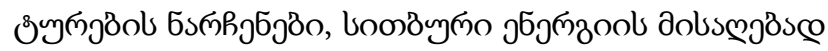

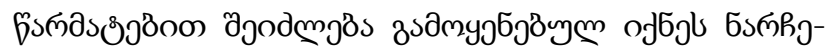

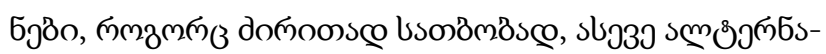

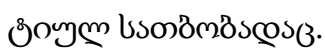

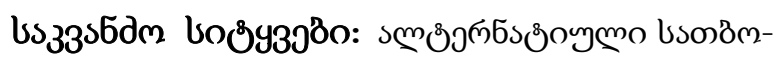

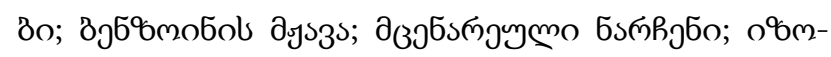

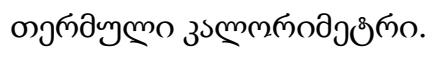

\section{gjlsзsмmo}

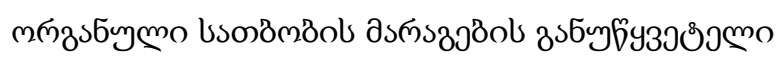

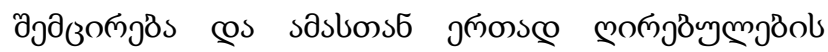




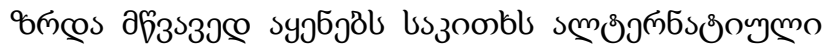

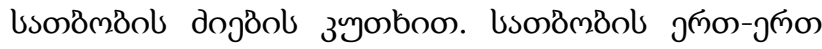

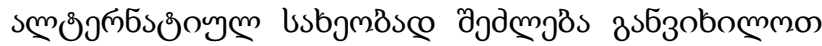

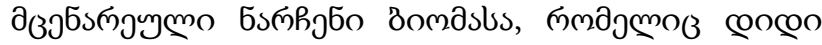

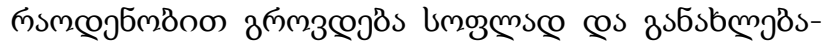

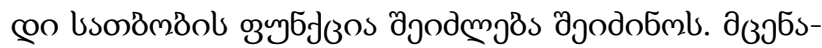

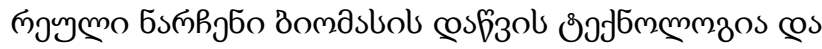

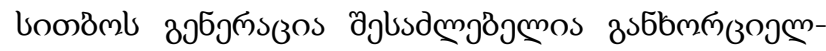

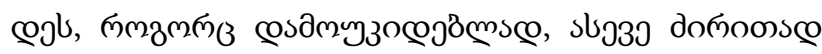

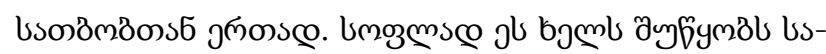

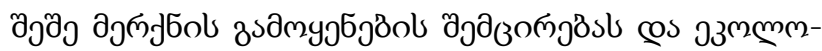

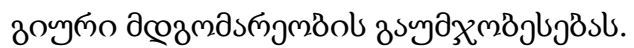

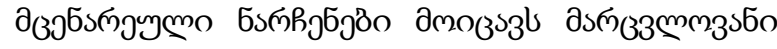

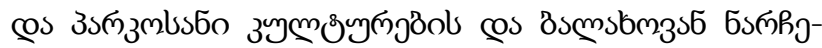

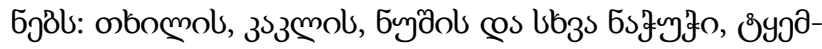

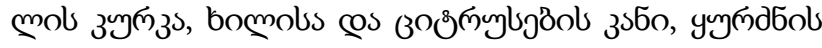

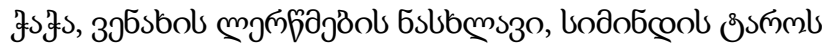

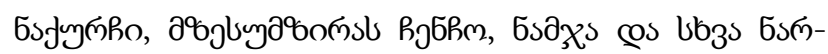

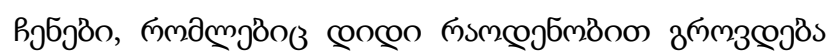

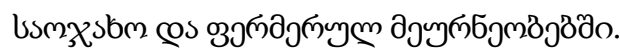

\section{donoonscon 5sfomo}

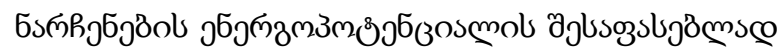

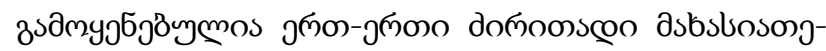

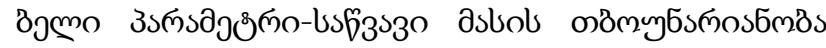

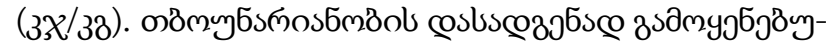

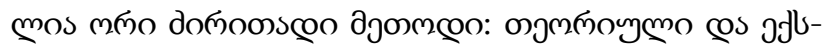

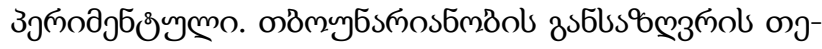

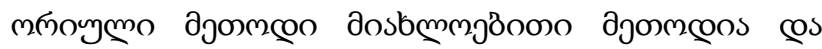

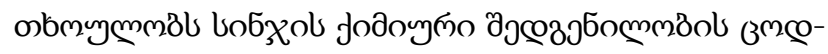

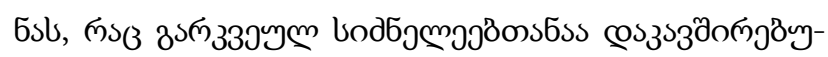

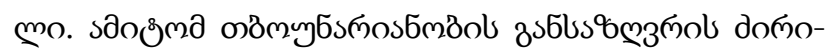

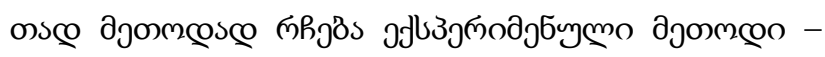

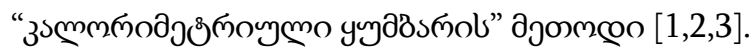

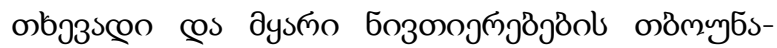

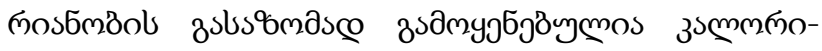

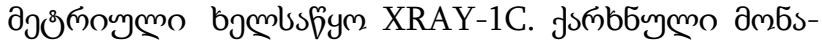

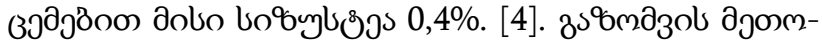

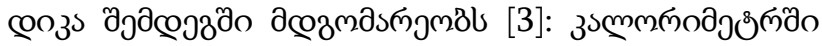

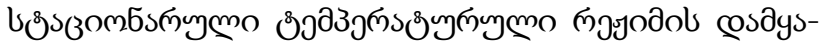

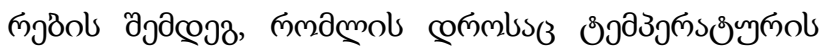

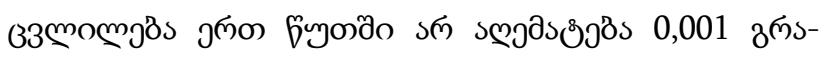

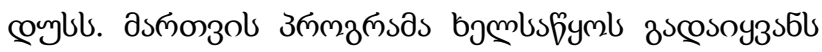

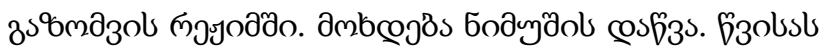

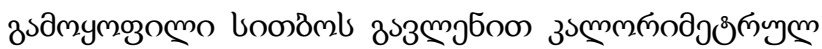

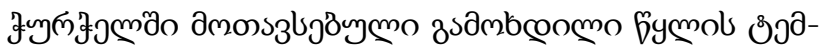

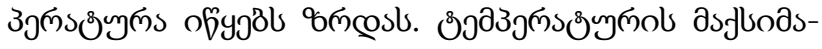

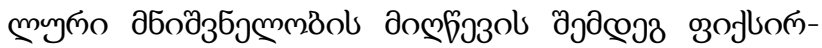

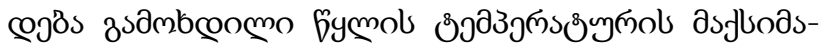

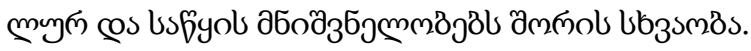

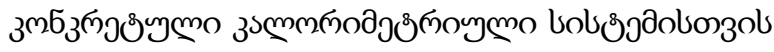

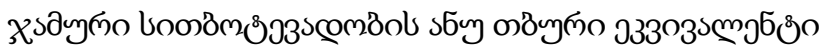

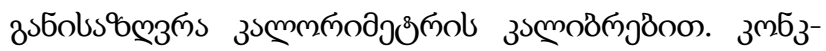

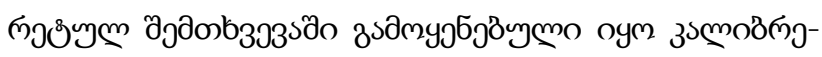

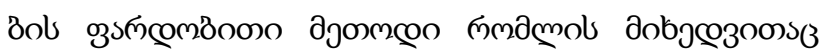

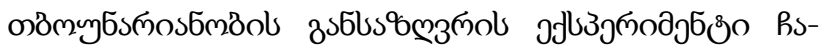

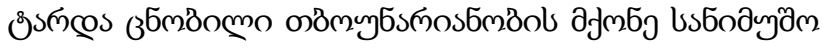

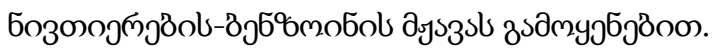

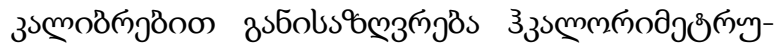

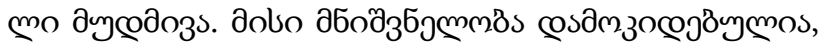

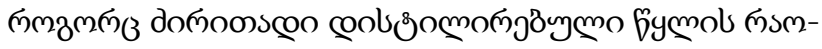

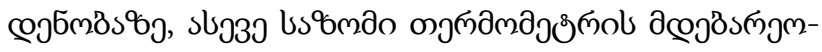

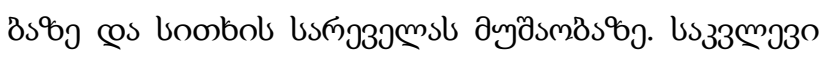

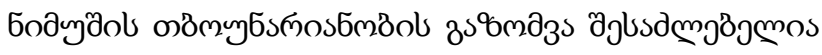

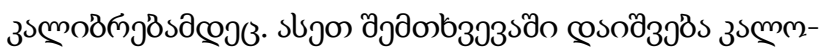

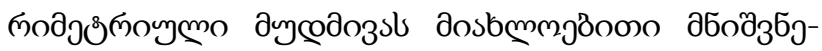

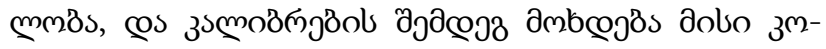

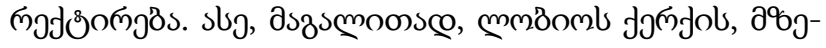




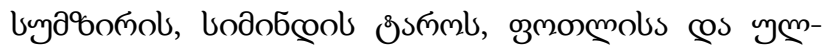

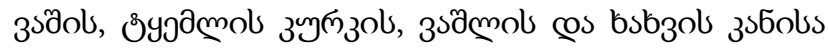

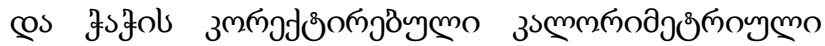

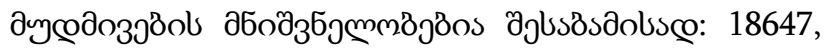
17495, 17213, 15778, 15778, 16352, 14344, 14344 cos

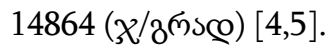

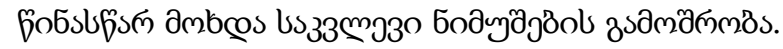

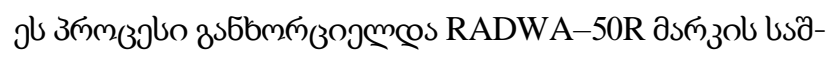

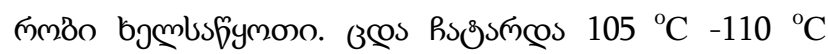

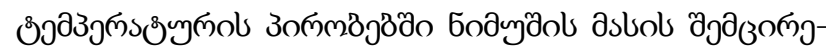

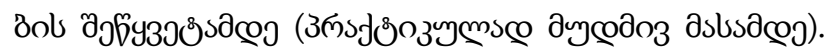

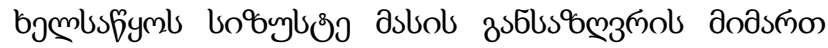

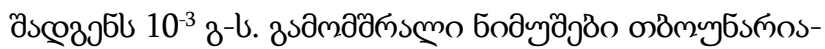

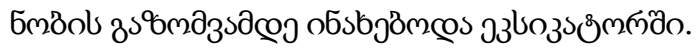

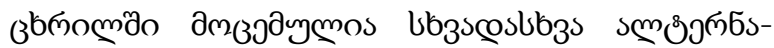

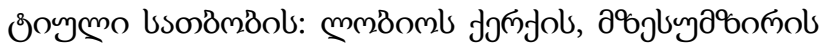

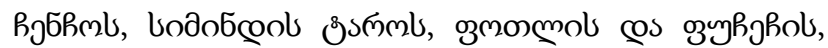

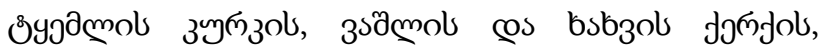

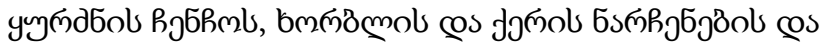

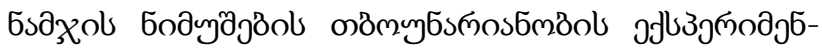

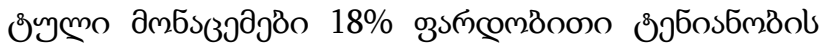

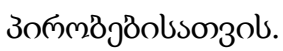

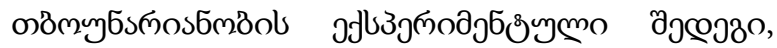

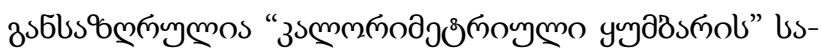

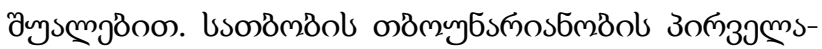

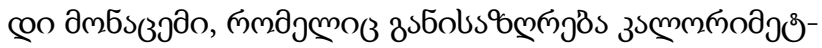

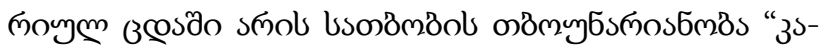

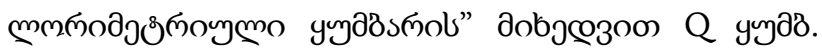

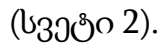

звпомо

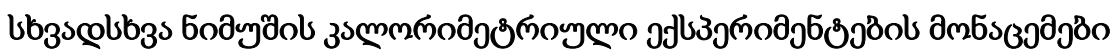

\begin{tabular}{|c|c|c|c|c|c|}
\hline № & 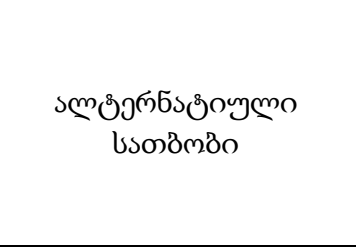 & 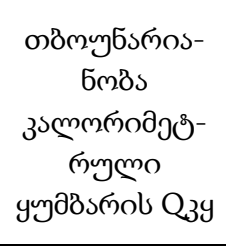 & 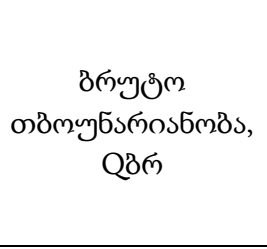 & 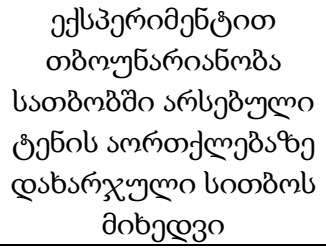 & 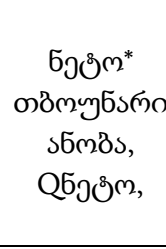 \\
\hline & 1 & 2 & 3 & 4 & 5 \\
\hline 1 & 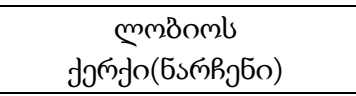 & 14783 & 14720 & 14268 & 12910 \\
\hline 2 & 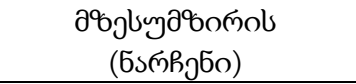 & 19354 & 19301 & 18848 & 17491 \\
\hline 3 & uodobcol ôs๘m & 18510 & 18447 & 17995 & 16637 \\
\hline 4 & 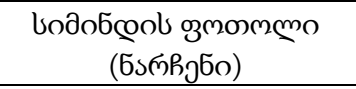 & 17428 & 17365 & 16912 & 15555 \\
\hline 5 & 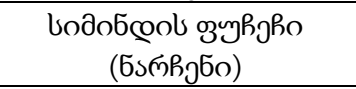 & 17692 & 17629 & 17176 & 15819 \\
\hline 6 & 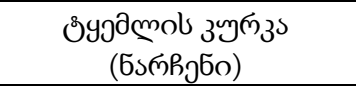 & 21767 & 21705 & 21252 & 19895 \\
\hline 7 & 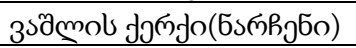 & 18287 & 18224 & 17772 & 16414 \\
\hline 8 & bsbzob jgn & 16392 & 16329 & 15876 & 14519 \\
\hline 9 & 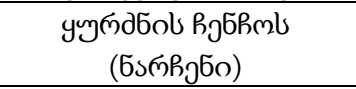 & 20640 & 20577 & 20125 & 18767 \\
\hline 10 & bмпв & 17585 & 17522 & 17070 & 15712 \\
\hline 11 & 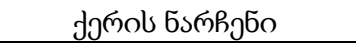 & 15793 & 15731 & 15279 & 13921 \\
\hline 12 & 5saxs & 16789 & 16726 & 16273,5 & 14916 \\
\hline
\end{tabular}




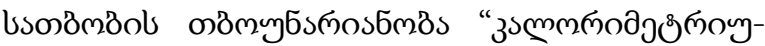

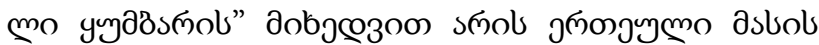

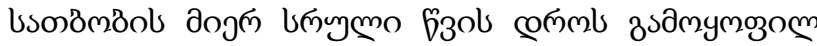

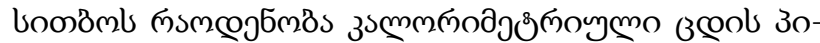

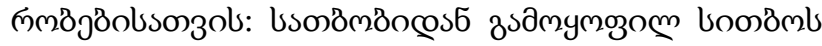

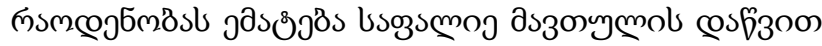

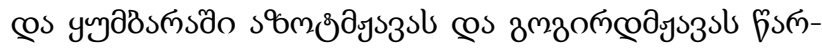

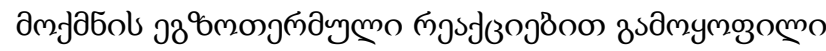

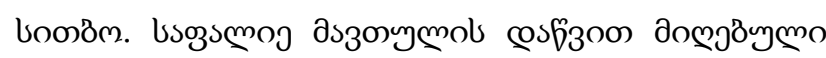

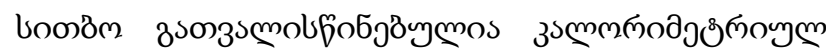

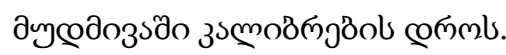

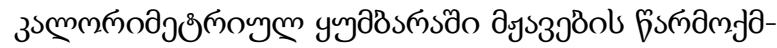

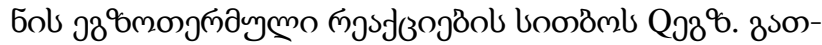

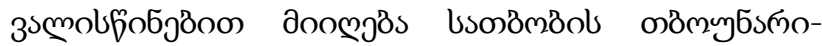

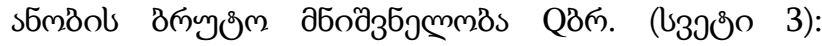

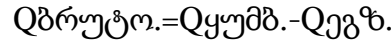

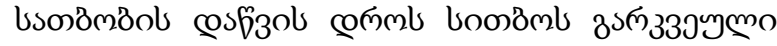

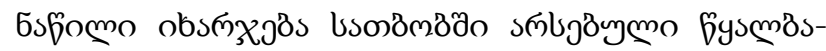

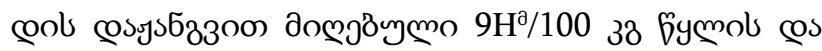

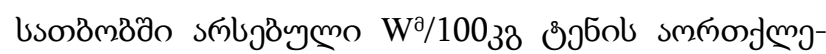

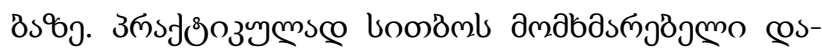

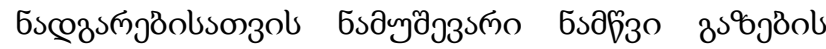

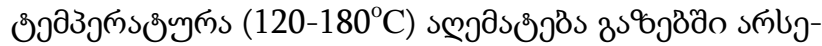

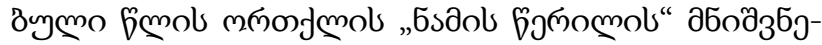

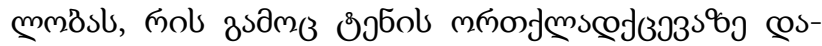

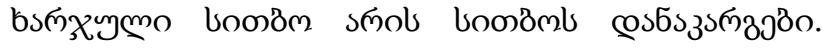

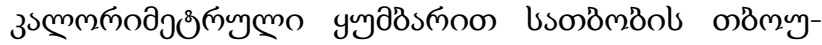

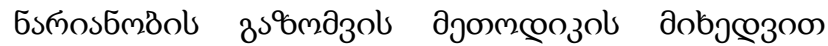

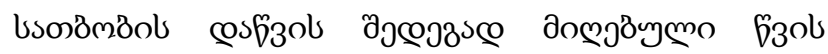

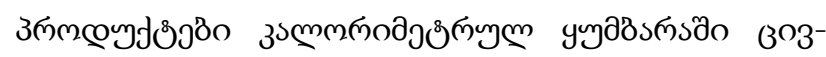

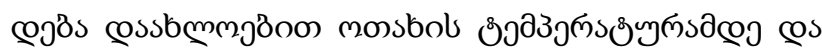

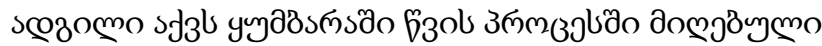

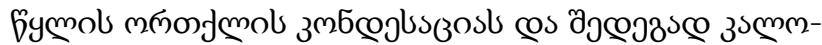

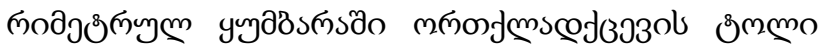

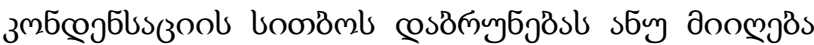

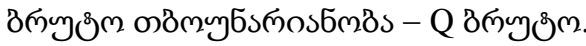

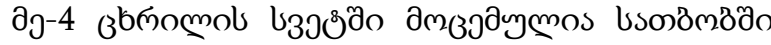

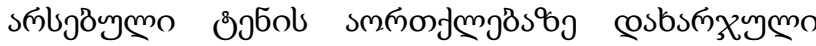

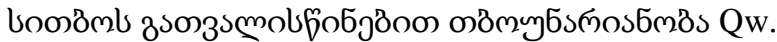

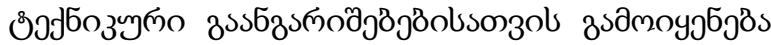

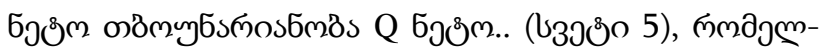

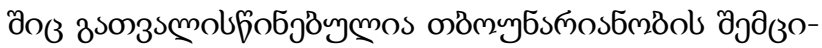

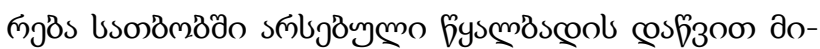

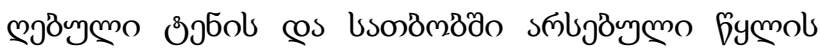

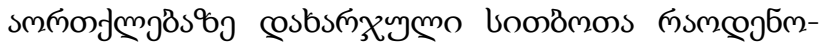
ठวอ̊๐:

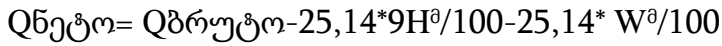

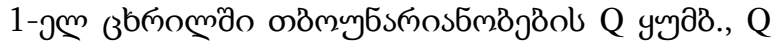

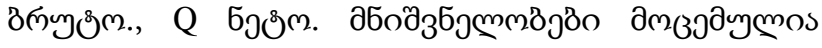

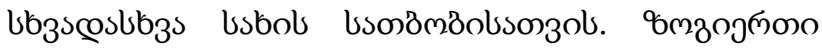

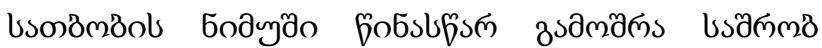

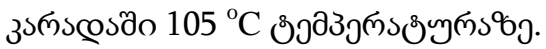

\section{costs336s}

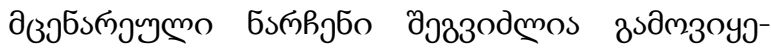

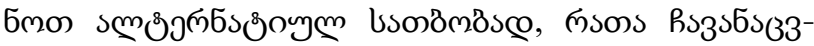

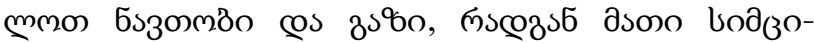

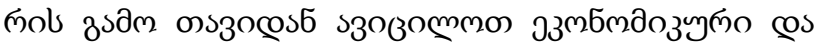
јб

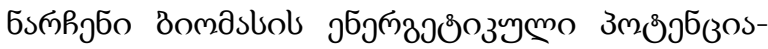

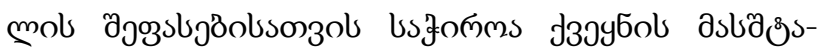

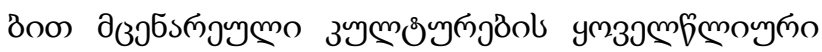

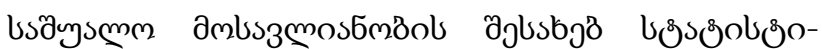

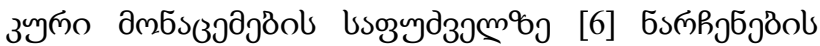

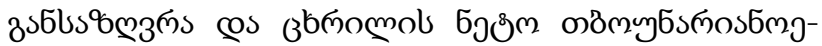

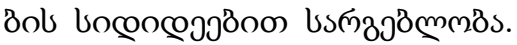




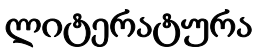

1. Kipshidze M., Jishkariani T., Arabidze G., Akhalaia G. Boiler installations. Tbilisi. 2000. (in Georgian).

2. Oleinik B.N. Exact calorimetry. M.: Izd. Standartov. 1973. (in Russian).

3. Lominadze S., Kipshidze M., Jishkariani T. Technical analysis of hydraulic and the exploitation test of steam

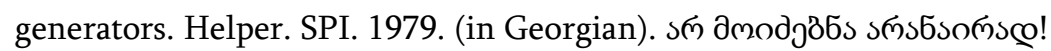

4. Kiguradze O., Japaridze O., Beridze G. Alternative fuels. "Energy". №1(85), 62-64pp. (in Georgian).

5. Kiguradze O., Japaridze O., Beridze G. Energy-efficient potential of plant residues biomass. "Energy". №1(85). 65-69 pp. (in Georgian).

6. Arabidze G., Arabidze N., Zarandia S. and others. The potential energy of the residual biomass in Georgia. Tilisi. 2015. (in Georgian).

\section{UDC 582.4}

SCOPUS CODE 2101

\section{Thermal study of plant residues biomass}

Otar Japaridze

Levan Akvlediani

Omar Kiguradze
Department of Thermal Energy and Energy Efficiency, Georgian Technical University, 75 M. Kostava str, 0160 Tbilisi, Georgia

E-mail: japaridzeotari@gmail.com

Department of Thermal Energy and Energy Efficiency, Georgian Technical University, 75 M. Kostava str, 0160 Tbilisi, Georgia

E-mail: samixada1995@gmail.com

Address.Department of Thermal Energy and Energy Efficiency, Georgian Technical University, 75 M. Kostava str, 0160 Tbilisi, Georgia

E-mail: kiguradzeomar@gmail.com

\section{Reviewers:}

A. Grigalashvili, Associate Professor, Faculty of Power Engineering and Telecommunication, GTU

E-mail: a.grigalashvili@gtu.ge

B. Chkhaidze, Associate Professor, Faculty of Power Engineering and Telecommunication, GTU

E-mail: bckaidze@yahoo.com

Abstract. The article reviews the possibilities for the usage of various plant residues as an alternative heating.

Experiments to measure biomass humidity and calorific value were carried out using a precision isothermal calorimeter MAY-1C. Benzoic acid (with 99.99\% purity) as standard material has been used for the calorimeter testing.

Experimental data of the highest calorific value of the samples were obtained, on the basis of which the lowest calorific values were calculated.

It was demonastrated that both the primary fuel and alternative fule can be used in Georgia's regions with various plant residues.

Key words: Alternative fuel; benzoic acid; isothermal calorimeter; plant residues.

ISSN 1512-0996

www.shromebi.gtu.ge 


\title{
UDC 582.4
}

SCOPUS CODE 2101

\section{Термическое исследование растительных отходов}

\author{
Отар Джапаридзе Департамент энергетики и энергоэффективности, Грузинский технический \\ университет, Грузия, 0160, Тбилиси, ул. М. Костава, 75 \\ E-mail: japaridzeotari@gmail.com \\ Леван Ахвледиани Департамент энергетики и энергоэффективности, Грузинский технический \\ университет, Грузия, 0160, Тбилиси, ул. М. Костава, 75 \\ E-mail: samixada1995@gmail.com \\ Омар Кигурадзе Департамент энергетики и энергоэффективности, Грузинский технический \\ университет, Грузия, 0160, Тбилиси, ул. М. Костава, 75 \\ E-mail: kiguradzeomar@gmail.com
}

\section{Рецензенты:}

А. Григалашвили, ассоциированный профессор факультета энегетики и телекомуникации ГТУ

E-mail: a.grigalashvili@gtu.ge

Б. Чхаидзе, ассоциированный профессор факультета энегетики и телекомуникации ГТУ

E-mail: bckaidze@yahoo.com

Аннотация. Рассмотрена перспектива применения разных сельскохозяйственных остатков в виде альтернативного топлива.

Эксперименты по измерению влажности и теплоспособности биомассы проведены с применением прецизионного изотермического калориметра МАY-1С. Для тестирования калориметра применено образцовое вещество - бензойная кислота (чистотой 99,99\%).

Получены экспериментальные данные высшей теплоспособности образцов , на основании которых рассчитаны величины самой низкой теплоспособности.

Показано, что в регионах Грузии, где сосредочены различные сельскохозяйственные остатки, можно с успехом применять как основное типливо, так и альтернативное.

Ключевые слова: альтернативное топливо; бензойная кислота; изотермический калориметр; сельскохозяйственные отходы.

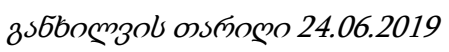

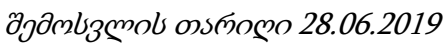

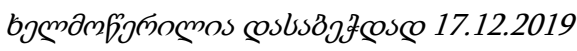

